\title{
Fine structure of critical opalescence spectra
}

\author{
M.Ya. Sushko \\ I.I. Mechnikov National University, 2 Dvoryanska Str., Odesa 65026, Ukraine \\ E-mail:mrs@onu.edu.ua
}

Received January 18, 2007

\begin{abstract}
The effect of the 1.5-scattering mechanism on the time and temperature behavior of the electric field autocorrelation function for the light wave scattered from fluids has been studied for the case where the order-parameter fluctuations obey the diffusion-like kinetics with spatially-dependent kinetic coefficient. The leading contributions to the relevant static correlation functions of the order-parameter fluctuations were obtained by using the Ginzburg-Landau model with a cubic term, and then evaluated with the use of the Gaussian uncoupling for many-point correlation functions and the Ornstein-Zernicke form for the pair correlation function. It is shown that the presence of the 1.5 -scattering effects in the overall scattering pattern may be detected in the form of a small but noticeable deviation from exponential decay of the total electric field autocorrelation function registered experimentally near the critical point. Obtained with the standard methods of analysis, the effective halfwidth of the corresponding spectrum can reveal a stronger temperature dependence and a multiplicative renormalization as compared to the halfwidth of the spectrum of the pair correlator.
\end{abstract}

PACS: 05 .70.Fh Phase transitions: general studies; 64.70.Fx Liquid-vapor transitions;

78.35.+c Brillouin and Rayleigh scattering; other light scattering.

Keywords: 1.5-scattering, critical opalescence, electric field autocorrelation function, halfwidth, multiplicative renormalization.

\section{Introduction}

Not counting multiple scattering effects, the intensity of critical opalescence spectra is thought to be proportional to the Fourier transform, with respect to the time and spatial variables, of the pair correlation function for the scalar order-parameter fluctuations $\delta \varphi[1]$. The central polarized component of these spectra is formed by the diffusion-like modes of $\delta \varphi$ and is usually believed to have a Lorentzian line shape with halfwidth $\Gamma(q)=\lambda(q) q^{2}$, where $\mathbf{q}$ is the change of the light wavevector due to scattering, and $\lambda(q)$ is the relevant kinetic coefficient (heat conduction or mutual diffusion).

Recently [2], we called attention to the fact that a so-called 1.5-scattering mechanism can become of importance as the critical point is approached, and presented experimental facts in support of this conclusion for the case of pure fluids [2-4]. In the present report, we scrutinize the role of the 1.5-scattering effects in the formation of critical opalescence spectra. In particular, we show that their presence in the overall scattering pattern can cause a small but noticeable deviation from exponential decay of the experimentally-measured electric field autocorrelation function. Calculated with the standard methods of analysis, the effective halfwidth $\Gamma_{\exp }$ of the corresponding spectrum can reveal a stronger temperature dependence in the form of a multiplicative renormalization, as compared to the halfwidth of the pair correlator spectrum.

\section{General structure of the critical opalescence spectrum}

To begin with, we analyze the critical opalescence spectrum by taking into account the effects of scattering by compact groups of fluctuations. A compact group is defined here as any group within which all the distances between the scattering centers are far less than the light wavelength in the medium. From a point of view of physicist, scattering by such groups is single, so that the overall single-scattering spectrum is expected to have a complex structure.

The electric field of a light wave propagating in an inhomogeneous fluid of permittivity $\varepsilon(\mathbf{r}, t)$ obeys the equation [1] 


$$
\Delta \mathbf{E}+\frac{1}{c^{2}} \frac{\partial^{2}}{\partial t^{2}} \varepsilon \mathbf{E}-\operatorname{grad} \operatorname{div} \mathbf{E}=0,
$$

where $c$ stands for the speed of light in vacuum. Suppose that the inhomogeneity of the fluid is caused by relatively slow fluctuations $\delta \varepsilon(\mathbf{r}, t)$ of the permittivity about its equilibrium value $\varepsilon_{0}, \varepsilon(\mathbf{r}, t)=\varepsilon_{0}+\delta \varepsilon(\mathbf{r}, t)$, which cause only small frequency changes $\Omega$ in the frequency $\omega$ of the scattered wave, as compared to the incident wave frequency $\omega_{0}:|\Omega|=\left|\omega-\omega_{0}\right|<<\omega_{0}$. For visible light, this condition is surely satisfied for the central line in the spectra of scattering from solutions and pure fluids $\left(\Omega \leq 10^{4 \div 5} \mathrm{~s}^{-1}\right)$, and even for the near wing of the Rayleigh line $\left(\Omega \leq 10^{10} \mathrm{~s}^{-1}\right)$. Then

$$
\left|\frac{\partial^{2} \varepsilon}{\partial t^{2}} \mathbf{E}\right|<<\left|\frac{\partial \varepsilon}{\partial t} \frac{\partial \mathbf{E}}{\partial t}\right|<<\left|\varepsilon \frac{\partial^{2} \mathbf{E}}{\partial t^{2}}\right|,
$$

and we can change to the equation

$$
\Delta \mathbf{E}+k^{2} \mathbf{E}-\operatorname{grad} \operatorname{div} \mathbf{E}=-k_{0}^{2} \delta \varepsilon \mathbf{E} .
$$

The form of (2) and that of the corresponding equation for the static case are formally the same, the time variable $t$ playing the role of a parameter. This means that after changing to the equivalent integral equation and applying the iteration procedure, the desired compact-group contributions to the scattered field can be extracted from every iteration step by following approach [2], based on analysis of the short-range singularities of the propagator in terms of the theory of generalized functions. As a result, the scattering intensity at frequency $\omega=\omega_{0}+\Omega$, which is proportional to the frequency Fourier transform of the autocorrelation function $I(t) \equiv<\mathbf{E}^{\prime}(\mathbf{R}, t) \cdot \mathbf{E}^{\prime *}(\mathbf{R}, 0)>$ for the scattered field, is determined mainly by the sum

$$
I(\mathbf{q}, \omega)=\sum_{n, m \geq 1} I_{n m}(\mathbf{q}, \Omega),
$$

where the term

$$
\begin{aligned}
& I_{n m}(\mathbf{q}, \Omega) \propto\left(-\frac{1}{3 \varepsilon_{0}}\right)^{n+m-2} \times \\
& \times \frac{1}{\pi} \operatorname{Re} \int_{0}^{+\infty} d t \int_{V} d \mathbf{r}<(\delta \varepsilon(\mathbf{r}, t))^{n}(\delta \varepsilon(0,0))^{m}>\mathrm{e}^{i \Omega t-i \mathbf{q r}}
\end{aligned}
$$

represents the contribution from pairs of compact groups of $n$ and $m$ fluctuations.

For the standard experiment geometry, where the scattered wave is registered in the plane perpendicular to the polarization vector of the incident wave, Eqs. (3) and (4) represent the intensity of polarized single scattering. It should be emphasized that it is the term $I_{11}$ alone that has been believed so far to form the critical opalescence spectra.

\section{Derivation of the 1.5-scattering spectrum for diffusion-like modes}

Now, we derive a formal expression for the contribution $I_{1.5}^{D}(q, t)$ from the 1.5 -scattering mechanism to $I(t)$, provided the fluctuations $\delta \varphi$ obey the diffusion-like kinetics. The desired contribution occurs in the terms with $n=1, m=2$ and $n=2, m=1$ in Eq. (3). In the time representation, those give

$$
\begin{aligned}
& I_{1.5}(q, t) \propto-\frac{1}{3 \varepsilon_{0}}\left(\frac{\partial \varepsilon}{\partial \varphi}\right)^{3} \times \\
& \times \int_{V} d \mathbf{r} \mathrm{e}^{-i \mathbf{q r}}\left[<\delta \varphi(\mathbf{r}, t)(\delta \varphi(\mathbf{0}, 0))^{2}>+<(\delta \varphi(\mathbf{r}, t))^{2} \delta \varphi(\mathbf{0}, 0)>\right] .
\end{aligned}
$$

For the diffusion-like modes, changing to the wavevector space, we can write

$$
\delta \varphi(\mathbf{r}, t)=\frac{1}{\sqrt{V}} \sum_{\mathbf{k}} \varphi_{\mathbf{k}}(0) \mathrm{e}^{-\lambda(k) k^{2} t+i \mathbf{k r}} .
$$

In terms of Eq. (6), the statistical averaging in (5) should be understood as averaging over the statistic of the initial values $\varphi_{\mathbf{k}}(0) \equiv \varphi_{\mathbf{k}}$. The need arises to use the third-order static correlation function $\left\langle\varphi_{\mathbf{k} 1} \varphi_{\mathbf{k} 2} \varphi_{\mathbf{k} 3}\right\rangle$, information of which is scanty at present. Nonetheless, some estimate becomes possible if we take into account the following facts [2-4] regarding the 1.5-scattering by pure fluids: 1) its contribution makes itself evident at a certain distance from the critical point, where nonlocal correlation between fluctuations is relatively weak; 2) it becomes considerable as a shift from the critical isochore occurs; 3 ) for the gas phase and, at least, densities $\rho<\rho_{c}$, $\rho_{c}$ being the critical one, its contribution is negative. Then, in view of the noninvariance of the fluid with respect to the transformation $\varphi(\mathbf{r}) \rightarrow-\varphi(\mathbf{r})$, let us suppose that it can be treated in terms of the Ginzburg-Landau Hamiltonian with a nonzero cubic term and a certain external field $h$ [5], the cubic and quartic terms being under the normal product sign [6]:

$H[\varphi]=$
$=\int_{V} d \mathbf{r}\left[\frac{a}{2} \varphi^{2}(\mathbf{r})+\frac{b}{2}(\nabla \varphi(\mathbf{r}))^{2}+\frac{c}{3} \varphi^{3}(\mathbf{r})+\frac{d}{4} \varphi^{4}(\mathbf{r})-h \varphi(\mathbf{r})\right]$.

The equilibrium value $\bar{\varphi}$ of the order-order parameter is expected to be uniform, so that the gradient term in Eq. (7) vanishes. Then

$$
\bar{\varphi}=\frac{1}{\sqrt{V}} \varphi_{\mathbf{k}=0}, \int_{V} d \mathbf{r} \delta \varphi(\mathbf{r})=0,
$$

and the fluctuation part of Eq. (7) can be represented as 
$H^{\prime}[\delta \varphi]=\int_{V} d \mathbf{r}\left[\frac{a^{\prime}}{2}(\delta \varphi)^{2}+\frac{b}{2}(\nabla \delta \varphi)^{2}+\frac{c^{\prime}}{3}(\delta \varphi)^{3}+\frac{d}{4}\left(\delta \varphi^{4}\right)\right]$,

with redefined coefficients $a^{\prime}$ and $c^{\prime}$. Near the critical isochore, we expect them both to have the structure close to $a^{\prime}=\alpha_{1} \tau+\alpha_{2} \omega$ and $c^{\prime}=\gamma_{1} \tau+\gamma_{2} \omega$, where $\alpha_{i}$ and $\gamma_{i}$ are constants, and the parameter $\omega=\left|V-V_{c}\right| / V_{c} \approx\left|\rho_{c}-\rho\right| / \rho$ describes the deviation from the critical isochore.

Treating the two last terms in Eq. (8) as a perturbation and using the Gaussian uncoupling, we find

$$
\begin{aligned}
& <\varphi_{\mathbf{k} 1} \varphi_{\mathbf{k} 2} \varphi_{\mathbf{k} 3}>= \\
& =-\frac{2 c^{\prime}}{k_{B} T \sqrt{V}} G\left(k_{1}\right) G\left(k_{2}\right) G\left(k_{3}\right) \delta_{\mathbf{k} 3,-\mathbf{k} 1-\mathbf{k} 2}+O\left(c^{\prime} d\right),
\end{aligned}
$$

where $k_{B}$ is the Boltzmann constant and $G(k) \equiv<\left|\varphi_{\mathbf{k}}\right|^{2}>$. Note that Eq. (9) resembles the convolution approximation for the tree-particle structure factor [7]. With Eq. (9), we find

$$
\begin{aligned}
& I_{1.5}^{D}(q, t) \propto \frac{2}{3 \varepsilon_{0}} \frac{c^{\prime}}{k_{B} T}\left(\frac{\partial \varepsilon}{\partial \varphi}\right)^{3} G(q) \mathrm{e}^{-\lambda(q) q^{2} t} \frac{1}{(2 \pi)^{3}} \times \\
& \times \int d \mathbf{k} G(k) G(|\mathbf{q}-\mathbf{k}|)\left[1+\mathrm{e}^{\lambda(q) q^{2} t-\lambda(k) k^{2} t-\lambda(|\mathbf{q}-\mathbf{k}|)|\mathbf{q}-\mathbf{k}|^{2} t}\right] .
\end{aligned}
$$

To advance, additional assumptions are needed. In particular, the coefficient $c^{\prime}$ can be evaluated if we accept the Ornstein-Zernicke form for $G(k)$, $G(k)=k_{B} T \chi /\left(1+k^{2} r_{c}^{2}\right)$, where $\chi$ is the susceptibility of the system. According to formulas (5) and (9), the integrated 1.5 -scattering intensity $I_{1.5}(q)=I_{1.5}(q, t=0)$ is then equal to

$$
I_{1.5}(q) \propto \frac{c^{\prime}}{3 \pi \varepsilon_{0}}\left(\frac{\partial \varepsilon}{\partial \varphi}\right)^{3} \frac{k_{B}^{2} T^{2} \chi^{3}}{q r_{c}^{4}\left(1+q^{2} r_{c}^{2}\right)} \arctan \left(\frac{q r_{c}}{2}\right) .
$$

In the hydrodynamic limit $q r_{c}<<1$, this expression must transform to the result [2], relating $I_{1.5}(q)$ to the third thermodynamic moment of the order-parameter fluctuations:

$$
I_{1.5}(q) \propto-\frac{2}{3 \varepsilon_{0}}\left(\frac{\partial \varepsilon}{\partial \varphi}\right)^{3} \widetilde{V}<(\Delta \varphi)^{3}>,
$$

where $\widetilde{V} \propto r_{c}^{3}$ is the volume of the region over which the correlators are averaged to pass to the corresponding thermodynamic moments. The proportionality coefficient in the latter relation can be recovered if we require that the computations of the contribution $I_{22}(q, t=0)$ with two different methods, the Gaussian model for $\delta \varphi$ and the macroscopic averaging [2], give identical results. Then $\widetilde{V}=12 \pi r_{c}^{3}$, which exceeds estimates [2].
The functional structure of the third moment was obtained earlier for the pure fluids $\left(\chi=\rho^{2} \beta_{T}, \beta_{T}\right.$ being the isothermal compressibility) that obey the van der Waals (see $[2,3])$ and Dieterici ([4]) equations. In the latter case, using the above $\widetilde{V}$, we have $\left(P_{c}\right.$ is the critical pressure): $c^{\prime}=-\left(\beta_{T}^{-1}+4 P_{c} \omega\right) / 3 \rho^{3}, \omega<<1$.

Since $I_{1.5}(q)$ is basically formed by the heat modes as the critical point is approached, $I_{1.5}(q) \approx I_{1.5}^{D}(q, t=0)$, this value can also be used for $c^{\prime}$ in Eq. (10). It also follows from Eq. (11) that for $\omega=0$ : 1) $I_{1.5}(q) \propto r_{c}$ as $q r_{c}<<1$, which is close to the scaling estimates $[2,6]$; 2) $I_{1.5}(q) \rightarrow 0$ as $q r_{c} \gg 1$, in accordance with the conformal invariance hypothesis [6].

The explicit form of $\lambda$ as a function of $q$ and temperature must be specified as well. To sufficient accuracy, we take $\lambda(q)=\lambda_{0} \tau^{v} \sqrt{1+q^{2} r_{c}^{2}}$, which is often considered to be a good interpolation formula for the entire critical region $[6,8]$ and has certain theoretical justifications [9-12] behind.

\section{Features of the 1.5-scattering spectrum}

We use the data for xenon to analyze the time and temperature behavior of the electric field autocorrelation function (10). We first assume a deviation $\omega=2 \cdot 10^{-1}$ from the critical isochore, where the correlation length exponent $v \approx 0.6$, take $\lambda_{0}=3 \cdot 10^{-8} \mathrm{~m}^{2} / \mathrm{s}$ for the value of the heat conduction coefficient far away from the critical point (as $\tau \rightarrow 1$ ), and use $q=2 \cdot 10^{7} \mathrm{~m}^{-1}$. The other date are (see [13]): $\varepsilon_{0}=1.3 ;(\rho \partial \varepsilon / \partial \rho)_{T}=0.33 ; r_{0}=2.2 \cdot 10^{-10} \mathrm{~m}$; $T_{c}=290 \mathrm{~K} ; P_{c}=5.83 \mathrm{MPa}$. The results of the analysis are as follows:

1. The relative magnitude $\left|I_{1.5}(q)\right| / I_{1}(q)$ of the integrated 1.5 -scattering intensity, as compared to the integrated intensity $I_{1}(q) \approx I_{11}^{D}(q, t=0)$ of the «standard» contribution

$$
I_{11}^{D}(q, t) \propto\left(\rho \frac{\partial \varepsilon}{\partial \rho}\right)^{2} \frac{k_{B} T \beta_{T}}{1+q^{2} r_{c}^{2}} \mathrm{e}^{-\lambda(q) q^{2} t},
$$

increases from 0.1 to as high as 0.35 as $\tau$ decreases from $1 \cdot 10^{-3}$ to $1 \cdot 10^{-4}$.

2. The time dependence of (10) deviates from exponential decay. Consequently, the 1.5 -scattering spectrum has a non-Lorentzian line shape. The ratio $\Gamma_{1.5} / \Gamma$ depends on the point chosen to calculate the slope of the $\ln I_{1.5}^{D}(q, t)$ versus $\Gamma t$ plot and increases as $\Gamma t$ decreases: for instance, if $\tau=1 \cdot 10^{-4}$, then $\Gamma_{1.5} / \Gamma \approx 1.1$ for $\Gamma t=0.6$ and $\Gamma_{1.5} / \Gamma \approx 1.4$ for $\Gamma t=0.2$.

3. For a fixed value of $\Gamma t$, the ratio $\Gamma_{1.5} / \Gamma$ increases as the critical point is approached.

Assuming now $\widetilde{V}=4 \pi r_{c}^{3} / 3$ [2], we see that $c^{\prime} \rightarrow 9 c^{\prime}$ and the above results hold for the $\omega=2.2 \cdot 10^{-2}$ isochore.

Thus, the 1.5 -scattering spectrum is represented by a line whose effective halfwidth $\Gamma_{1.5}$ exceeds $\Gamma$; the ratio 
$\Gamma_{1.5} / \Gamma$ gets greater as we shift toward (a) the region of higher frequencies and/or (b) the critical point. Contributing, in addition, negatively to the total scattering intensity, the 1.5 -scattering can manifest itself in the form of an additional narrowing of the registered spectrum. This narrowing can be interpreted as a stronger temperature dependence of the registered halfwidth $\Gamma_{\exp }$ as compared to that of $\Gamma$, thus necessitating the introduction of a new critical exponent $\phi$ and a multiplicative coefficient $R$.

\section{Effective halfwidth of the experimentally- registered spectrum}

The temperature behavior of $\Gamma_{\exp }$ is determined as follows. For different fixed values of $\tau$, we plot $\ln \left[I_{11}^{D}(q, t)+I_{1.5}^{D}(q, t)\right]$ as a function of $\Gamma t$ and then calculate the ratio $\Gamma_{\exp } / \Gamma$ as the slope of the straight line approximating this plot on a fixed segment, say $\Gamma t \in[0.2,0.6]$. Then we represent $\Gamma_{\exp }$ in the form $\Gamma_{\exp }=R \tau^{\phi} \Gamma$, which is equivalent to

$$
\log \left(\Gamma_{\exp } / \Gamma\right)=\log R+\phi \log \tau,
$$

and determine the values $\phi$ and $\log R$ as, respectively, the slope and the intercept of the line tangent to the $\log \left(\Gamma_{\exp } / \Gamma\right)$ versus $\log \tau$ plot.

The results for the temperature region $\tau=5 \cdot 10^{-5}-6 \cdot 10^{-3}$ are shown in Fig. 1. In the region $\tau>1 \cdot 10^{-3}$, where the 1.5 -scattering is inessential, $\phi \rightarrow 0$ and $R \rightarrow 1$. For the interval $\tau=5 \cdot 10^{-5}-4 \cdot 10^{-4}$, where $\Gamma_{\exp }$ reduces to $90 \%$ of $\Gamma$, we find $\phi \approx 0.035$ and $R \approx 1.31$ (the straight line in

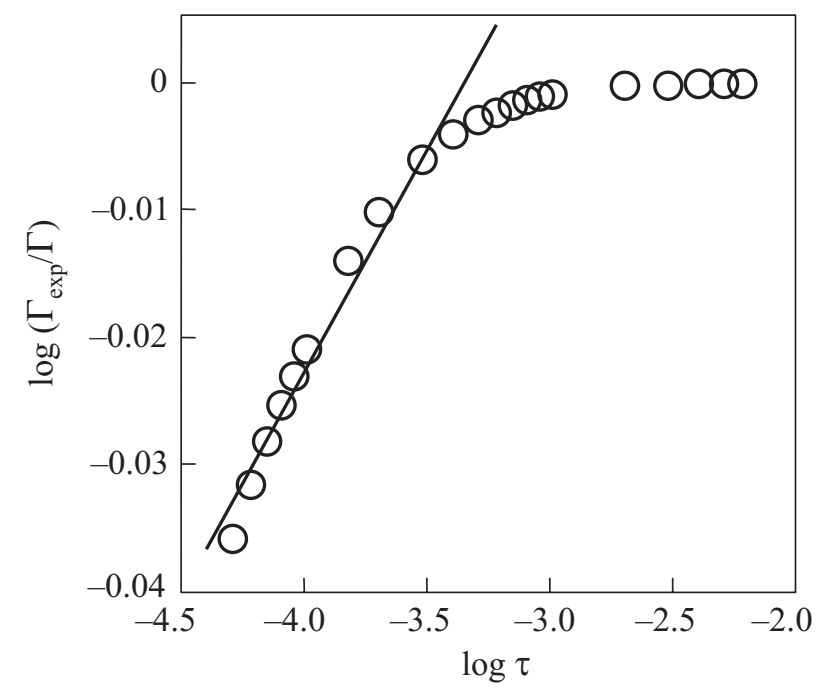

Fig. 1. $\log \left(\Gamma_{\exp } / \Gamma\right)$ as a function of $\log \tau$ for $\tau=5 \cdot 10^{-5}-6 \cdot 10^{-3}$, recovered through segment $\Gamma t \in[0.2,0.6]$.
Fig. 1); the use of segment $\Gamma t \in[0.1,0.6]$ results in $\phi \approx 0.05$ and $R \approx 1.48$. These estimates are consistent with experiment (see [14]).

\section{Conclusion}

The above analysis reveals that the spectrum of molecular light scattering from fluids near the critical point has a complex structure. Besides the pair correlation function of the order-parameter fluctuations, it is also contributed to by the 1.5 -scattering mechanism. The latter can manifest itself in the form of a stronger temperature dependence of the experimentally-measured spectral halfwidth, as compared to that of the pair correlation function. The effect is usually attributed to other factors, such as the presence of large background parts in the kinetic coefficients [15] or multiplicative renormalization of these coefficients [16] in the vicinity of the critical point.

In the case of pure fluids, the 1.5 -scattering effects come into play whenever a shift from the critical isochore occurs, planned or accidental. Since such a shift is greatly favored by the gravity effect, taking the 1.5 -scattering contribution into account becomes of crucial importance for correct processing and interpretation of experimental data.

1. L.D. Landau and E.M. Lifshitz, Electrodynamics of Continuos Media, Pergamon Press, Oxford (1984).

2. M.Ya. Sushko, Zh. Eksp. Teor. Fiz. 126, 1355 (2004).

3. M.Ya. Sushko, Condens. Matter Phys. 9, 37 (2006).

4. M.Ya. Sushko, Ukr. J. Phys. 51, 758 (2006).

5. L.D. Landau and E.M. Lifshitz, Statistical Physics, Pergamon Press, Oxford (1984).

6. A.Z. Patashinskii and V.L. Pokrovskii, Fluctuation Theory of Phase Transitions, Pergamon Press, Oxford (1984).

7. I.O. Vakarchuk, Introduction into the Many-Body Problem, Lviv Univ. Press, Lviv (1999).

8. R. Perl and R.A. Ferrell, Phys. Rev. Lett. 29, 51 (1972).

9. B.J. Ackerson, C.M. Sorensen, R.C. Mockler, and W.J. O'Sullivan, Phys. Rev. Lett. 34, 1371 (1975).

10. C.M. Sorensen, B.J. Ackerson, R.C. Mockler, and W.J. O'Sullivan, Phys. Rev. A13, 1593 (1976).

11. N.P. Malomuzh and M.Ya. Sushko, Zh. Eksp. Teor. Fiz. 89, 435 (1985).

12. M.Ya. Sushko, Optika i Spektr. 73, 1175 (1992).

13. N.J. Trappeniers, A.C. Michels, H.M.J. Boots, and R.H. Huijser, Physica A (Amsterdam) 101, 431 (1980).

14. M.A. Anisimov, Critical Phenomena in Fluids and Liquid Crystals, Nauka, Moscow (1987).

15. J.V. Sengers, in: Critical Phenomena. Int. School of Physics «Enrico Fermi», LI Course, M.S. Green (ed.), Academic Press, New York (1971), p. 445.

16. T. Ohta, Prog. Theor. Phys. 54, 1566 (1975). 